\title{
Smoking Experimentation, Moderate-to-Vigorous Physical Activity, and Associated Psychosocial and Demographic Correlates among Mexican Origin Youth
}

\author{
Anna V. Wilkinson ${ }^{1 *}$, Melissa H. Stigler ${ }^{1}$, Nnenna L. Okeke ${ }^{1}$, and Margaret R. Spitz ${ }^{2}$ \\ ${ }^{1}$ University of Texas School of Public Health, Austin Regional Campus, and Michael \& Susan Dell Center for \\ Healthy Living, Austin, TX, USA and ${ }^{2}$ Department of Molecular and Cellular Biology, Baylor College of \\ Medicine, Houston, TX, USA
}

\begin{abstract}
Moderate-to-vigorous physical activity (MVPA) may deter adolescents from smoking initiation and psychosocial risk and protective factors associated with both physical activity and smoking experimentation may play a role. This paper examines such psychosocial factors across four groups of adolescents identified by crossing smoking experimentation (yes/no) with completion of regular MVPA (yes/no). In 200809, 1,098 Mexican origin youth (553 girls and 546 boys, mean age 14.3 years [SD=1.04]) provided data on smoking behavior, physical activity, alcohol use, acculturation, peer and parental smoking, BMI, body image, smoking outcome expectations, anxiety, subjective social status (SSS), body image, and sensationseeking tendencies. We conducted Pearson's $\chi^{2}$ tests to examine associations between a) MVPA and smoking and $b$ ) demographic variables and the four groups identified by crossing smoking experimentation with MVPA. Next, adjusting for age and gender, we completed analysis of covariance to examine differences in psychosocial factors across the four groups. In our sample, $22.4 \%$ had experimented with cigarettes, and 29.3\% completed adequate MVPA. Both behaviors were more prevalent among boys. Although not statistically significantly higher, $32.9 \%$ of experimenters completed adequate MVPA compared to 28.3\% among non-experimenters. Experimenters who complete adequate MVPA reported the highest levels of thrill and adventure seeking, while those who completed inadequate MVPA reported lower SSS and more body image concerns than non-experimenters $(p<0.05$ for all). Our findings highlight an opportunity to address MVPA as an alternative to smoking among Mexican origin youth with high thrill and adventure sensation-seeking tendencies, $82 \%$ of whom have experimented and $69 \%$ of whom completed MVPA.
\end{abstract}

Keywords: moderate-to-vigorous physical activity, smoking behavior, shared psychosocial correlates, Mexican origin youth.

\section{INTRODUCTION}

The risk of smoking initiation is highest during adolescence [1,2]. It is also a time when patterns of physical activity are likely to change, becoming less frequent than in pre-adolescence [3]. A decrease in physical activity may be particularly common among teen smokers, who are generally less physically active than their non-smoker counterparts [4]. Smoking and physical activity behaviors during adolescence are likely to track into adulthood, impacting risk for chronic disease and various cancers [5,6]. Evidence suggests that regular moderate-to-vigorous physical activity (MVPA), defined as being physically active for 60 minutes on at least 5 of the 7 previous days, may deter adolescents from both the initiation and continuation of smoking [7]. However to intervene

"Address correspondence to Anna V. Wilkinson, University of Texas School of Public Health, Austin Regional Campus, 1616 Guadalupe, Suite 6.300, Austin, TX 78701, USA; Tel: 512-391-2528; Fax: 512482-6185; E-mail: anna.v.wilkinson@uth.tmc.edu effectively, physical activity programs would require knowledge of how psychosocial risk factors differ across groups of youth with different levels of physical activity and cigarette experimentation experience, so as to identify characteristics of youth most and least at risk for these life-long health-related behaviors.

Engaging in physical activity may be connected to teens' perceptions of the activity and the feelings the activity elicits [8]. Our previous research on Mexican origin youth suggests similar motivations for smoking [9-11]. Those who experiment with cigarettes are likely to exhibit sensation-seeking tendencies, higher anxiety, worse body image, and lower subjective social status (SSS) [9-11]. They are also more likely to expect positive outcomes from smoking [9]. Smoking experimenters are also more likely to experiment with other substances, such as alcohol and other drugs $[12,13]$. Of note, higher levels of sensation-seeking tendencies, lower SSS, poor body image, and lower 
anxiety among Mexican origin youth are also associated with regular MVPA $[14,15]$.

Most research, to date, has examined Hispanic youth as a group, but the study of the subgroup of Mexican origin youth is important because people of Mexican origin represent the largest and most rapidly growing subgroup of Hispanics in the United States [16]. Hispanics are the largest and most rapidly growing ethnic group in the United States and are from diverse ethnic backgrounds that are culturally distinct from each other [16-18]. We focus on the subgroup of Mexican origin youth in order to identify unique risk factors associated with a single subgroup of Hispanics. Hispanic youth in Texas, of whom Mexicans represent the majority, report higher rates of cigarette experimentation and lower rates of adequate physical activity compared to youth of other ethnic backgrounds [19]. For these reasons, studying smoking behavior and physical activity in this population with the goal of preventing smoking initiation, is timely and warranted.

Physical activity and smoking during adolescence occurs within a social context that can influence adolescents to engage in these behaviors. Mexican origin adolescents are more likely to try cigarettes if they have family members or friends who smoke [20,21]. Furthermore, physical activity was found to be most common among the least and most acculturated youth, compared to those of moderate acculturation [10]. No studies, however, have explored how these two behaviors and their intrapersonal and social environmental risk factors intersect among Mexican origin youth.

The acknowledgement of the association between physical activity and smoking has yielded intervention efforts in which the former is used, at times effectively, as part of smoking cessation programs for both adults and teens [22,23]. A similar approach may be beneficial to prevent smoking experimentation and continuation among adolescents. Therefore, in this paper we examine psychosocial differences among four groups of Mexican origin adolescents: physically active cigarette experimenters, physically active nonexperimenters, non-physically active cigarette experimenters, and non-physically active nonexperimenters in order to identify characteristics of youth most and least at risk for tobacco use.

\section{METHODS}

\section{Study Design}

Participants in the current study are part of a prospective study of smoking behavior, and were recruited from a large population-based cohort of Mexican American households in the Houston metropolitan area launched in 2001 and maintained by the Department of Epidemiology at The University of Texas M.D. Anderson Cancer Center. Household members were recruited into the Mano-a-Mano Mexican American Cohort Study (MACS) using door-to-door recruitment, probability random-digit dialing, intercepts, and networking approaches. Analysis of the pooled recruitment methods revealed no significant differences between the sample and the census tract from which they were drawn, with respect to language preference, country of origin, years living in the US, and household income [24]. A detailed description of the cohort recruitment methods has been previously published [25].

Three thousand households with adolescents between the ages of 11 and 13 years were identified from the MACS database. Of the first 1,425 contacted, just over $90 \%$ of parents and legal guardians $(N=1,328)$ provided written informed consent while the child provided written informed assent prior to enrolling in the study in 2005-06. The Institutional Review Board at The University of Texas M.D. Anderson Cancer Center granted approval for both the MACS and this study.

\section{Data Collection}

A personal digital assistant (PDA) survey was administered in 2005-06 and again in 2008-09, via personal interviews in the participants' homes, following data collection procedures that have been previously published [20]. Briefly, after a 5minute interview in which demographic information was obtained, participants privately completed a survey on a PDA. Participants completed the survey in either English or Spanish and received a gift certificate (\$25) after each interview. 


\section{Measures}

\section{Outcome Measure}

Our main outcome variable was a four-level categorical variable based on reported MVPA level and smoking experimentation status. The four categories were: adequate MVPA and nonexperimenter; adequate MVPA and experimenter; inadequate MVPA and non-experimenter; and finally inadequate MVPA and experimenter.

Smoking experimentation was assessed by the PDA survey questions, "Have you ever smoked a whole cigarette?" and "Have you ever tried a cigarette, even a puff?" A response of yes to either item indicated experimentation. For this analysis, participants who reported no experience with tobacco were considered non-experimenters, and coded as ' 0 '. Participants who reported having tried cigarettes, at least once, were considered experimenters and were coded as ' 1 '.

MVPA was assessed using the following two survey items: "Think about the activities you do at school, but not in PE. On how many days, of the past 7 , did you exercise or participate in physical activity for at least 60 minutes per day?" and "Think about activities you do in your community or at home. On how many days, of the past 7 , did you exercise or participate in physical activity for at least 60 minutes per day?" both of which were adapted from the Youth Risk Behavioral Surveillance System [26]. To be consistent with 2005 guidelines for MVPA, using both variables and regardless of context, participants who reported being physically active for at least 60 minutes on at least 5 days, out of the previous 7 , were considered to have adequate MVPA and coded as ' 0 '. Others were considered to have inadequate MVPA and were coded as ' 1 '.

\section{Behavioral and Psychosocial Covariates}

The behavioral and psychosocial covariates are described in Table 1.

\section{Demographic Covariates}

Participants reported their gender as either male or female, and their country of birth as either the United States or Mexico. Age was entered as a continuous variable. Socio-economic status (SES) was assessed using highest parental educational attainment rather than household income. Both are valid markers of SES, but more than $40 \%$ of parents did not report income, while the majority reported educational attainment [27]. SES was categorized as less than high school, high school/General Educational Development equivalency, or more than high school. Body Mass Index (BMI) was calculated using the height and weight measurements taken by trained interviewers at follow-up in 2008-09. Seca scales and stadiometers were used following a standard protocol.

All variables in the current analysis were assessed at follow-up in 2008-09, with the exception of parental education, which was assessed when the parent enrolled in the MACS.

\section{Statistical Analyses}

For descriptive purposes, we calculated frequencies for gender, country of birth, parental education, MVPA, and smoking experimentation, while for age and the psychosocial correlates of interest, we calculated means and standard deviations. We conducted Pearson's $\chi^{2}$ tests to examine the association between MVPA and smoking experimentation, as well as the associations between gender, country of birth, and parental educational attainment, and the four-level categorical variable combining MVPA and smoking experimentation status. We used analysis of variance (ANOVA) to examine unadjusted mean differences in age by the four-level categorical variable. We used analysis of covariance (ANCOVA) to examine mean differences in the psychosocial correlates by the four-level categorical variable, adjusting for age and gender. Post-hoc comparisons of significant mean differences of the psychosocial correlates were completed and statistical significance was assessed using a Bonferroni adjustment to correct for the multiple comparisons.

\section{RESULTS}

Table 2 depicts the frequencies, means, and standard deviations of participant demographic characteristics, outcome variables, and the correlates of interest. The current analyses are based on 1,098 participants, or $95 \%$ of the 1,154 participants who provided data at follow-up in 2008-09, 


\section{Table 1:Psychosocial and Behavioral Traits Examined in Relation to Smoking Experimentation} and Regular Completion of Moderate-to-Vigorous Physical Activity

\begin{abstract}
Acculturation
[41]

Assessed using four items that ascertain language used when reading, speaking at home, speaking with friends, and thinking. Responses are made on a five-point scale ranging from 'only Spanish' to 'only English'. Acculturation scores reflect the mean of the four items. Based on our data, the scale demonstrates excellent internal reliability (alpha=0.92).
\end{abstract}

from friends and

family

Positive outcome expectations [42]

Trait anxiety [44]

Subjective social status [46]

Body Shape Questionnaire [47]

Sensation Seeking Scale [48]

Team sports participation [26]

Alcohol Use [26]

Parental influence on smoking was assessed using two questions: 'Does your father/ stepfather smoke?' and 'Does your mother/stepmother smoke?' The variable reflects if none, one, or both parents smoke. Similarly, peer influence was assessed by asking: 'how many of your three closest friends smoke?' The variable reflects if none, one, or two or more friends smoke.

Outcome expectations are the beliefs about the positive or negative consequences of engaging in a specific behavior [43]. Assessed using seven items. Responses for each item are made on a four-point scale that ranges from 'strongly disagree' to 'strongly agree'. Positive outcome expectation scores reflect the mean of the seven items, ranging from 1 to 7. The scale demonstrates very good internal reliability based on our data (alpha=0.88).

Trait anxiety refers to a relatively stable response or proneness to anxiety [40]. Assessed using 20 items. Response options range from 'not at all' to 'very much so' on a 4-point scale. Trait anxiety scores reflect the mean of the 20 items, ranging from 20 to 80 . The scale has been validated in US Spanish-speaking samples; based on our data, the scale demonstrates very good internal reliability (alpha=0.86) [45].

Participants place themselves on a 10-rung ladder, with higher rungs representing more positive perceptions of social status as it relates to grades in school, number of friends, and proficiency at sports.

Body image was assessed using 10 items. Response options included 'no', 'not sure', or 'yes'. Each 'yes' was added to create a composite score, ranging from 0 to 10 ; higher scores reflect greater concern with body image. The scale demonstrates good internal reliability based on our data (alpha $=0.70$ ).

Sensation seeking is a personality trait that takes the form of seeking novel, varied, complex, and intense sensations and experiences and the willingness to take physical, social, legal, and financial risks in order to experience this sensation [49]. Drug and alcohol seeking (DAS), social disinhibition, and thrill and adventure seeking (TAS) were assessed using subscales from a larger 26-item sensation seeking scale. Based on data from our participants, each scale demonstrated good internal reliability $\left(\right.$ alpha ${ }^{\mathrm{DAS}}=0.72$; alpha $^{\text {social }}$ disinhibition $=0.68$; alpha ${ }^{\mathrm{TAS}}=0.81$ ).

School team sports and community team sports participation were assessed using the following two items, both of which were adapted from the 2005 Youth Risk Behavioral Surveillance System (YRBSS): "Think about the activities you do at school but not during P.E. During the past 12 months, on how many sports teams did you play?" and "Think about the activities you do in your community or at home. During the past 12 months, on how many sports teams did you play?" Responses to both items were summed to reflect the total number of sports teams on which the participant played in the past 12 months.

To assess alcohol use, participants were asked "During your life, on how many days have you had at least one drink of alcohol?" The item is taken directly from the 2005 YRBSS: Participants who reported 0 days of alcohol use were considered never-drinkers, while those who reported having tried alcohol at least once were considered ever-drinkers. and for whom there were complete data for the variables of interest. The sample was nearly evenly split by gender, and ages ranged from 1217 with a mean of 14.32 years (SD=1.04). USborn participants comprised nearly three-fourths of the sample, and $65.3 \%$ had parents with less than a high school education. Overall, $22.4 \%$ of participants had experimented with cigarettes and $29.3 \%$ completed adequate MVPA. 
Table 2: Participant Characteristics by Demographic Factors, Behaviors and Psychosocial Factors $(\mathrm{N}=1,098)$

\begin{tabular}{|c|c|}
\hline Demographic Factors & $\mathbf{N}(\%)$ \\
\hline \multicolumn{2}{|l|}{ Gender } \\
\hline Female & $553(50.4)$ \\
\hline Male & $545(49.6)$ \\
\hline \multicolumn{2}{|l|}{ Age (Years) } \\
\hline Mean (SD) & $14.32(1.04)$ \\
\hline \multicolumn{2}{|l|}{ Range: $12-17$} \\
\hline \multicolumn{2}{|l|}{ Country of Birth } \\
\hline Mexico & $286(26.0)$ \\
\hline US & $812(74.0)$ \\
\hline \multicolumn{2}{|l|}{ Parental Education } \\
\hline$<\mathrm{HS}$ & $717(65.3)$ \\
\hline HS & $192(17.5)$ \\
\hline$>\mathrm{HS}$ & $189(17.2)$ \\
\hline \multicolumn{2}{|l|}{ Behaviors } \\
\hline \multicolumn{2}{|l|}{ MVPA } \\
\hline Inadequate & $776(70.7)$ \\
\hline Adequate & $322(29.3)$ \\
\hline \multicolumn{2}{|l|}{ Smoking Experimentation } \\
\hline Non-Experimenter & $852(77.6)$ \\
\hline Experimenter & $246(22.4)$ \\
\hline Psychosocial Factors & Mean (SD) \\
\hline \multicolumn{2}{|l|}{ Acculturation } \\
\hline Range: $1-5$ & $3.50(0.72)$ \\
\hline \multicolumn{2}{|l|}{ No. Parents who Smoke } \\
\hline Range: $0-2$ & $0.37(0.56)$ \\
\hline \multicolumn{2}{|l|}{ No. Friends who Smoke } \\
\hline Range: 0-3 & $0.34(0.74)$ \\
\hline \multicolumn{2}{|l|}{ BMI } \\
\hline Range: $14.57-58.00$ & $25.03(6.33)$ \\
\hline \multicolumn{2}{|l|}{ Community Team Sports } \\
\hline Range: 0-7 & $1.49(1.67)$ \\
\hline \multicolumn{2}{|l|}{ School Team Sports } \\
\hline Range: 0-7 & $1.71(1.71)$ \\
\hline \multicolumn{2}{|l|}{ Alcohol Use } \\
\hline Range: $0-1$ & $0.29(0.46)$ \\
\hline \multicolumn{2}{|c|}{ Smoking Outcome Expectations } \\
\hline Range: 1-7 & $1.33(0.48)$ \\
\hline \multicolumn{2}{|l|}{ Trait Anxiety } \\
\hline Range: $20-70$ & $38.32(9.44)$ \\
\hline \multicolumn{2}{|l|}{ Subjective Social Status } \\
\hline Range: $1-10$ & $7.75(1.49)$ \\
\hline \multicolumn{2}{|l|}{ Body Image } \\
\hline Range: 1-10 & $1.83(2.25)$ \\
\hline
\end{tabular}

Drug \& Alcohol Seeking

Range: 0-7

$1.13(1.56)$

Social Disinhibition

Range: 0-7

$3.27(1.93)$

Thrill \& Adventure Seeking

Range: 0-12

$6.85(3.30)$

SD: standard deviation. Participants who reported being physically active for at least 60 minutes on at least 5 days were considered to have adequate MVPA; all others were considered to have inadequate MVPA. Participants who responded yes to either "Have you ever smoked a whole cigarette?" or "Have you ever tried a cigarette, even a puff?" were considered experimenters; participants who answered no to both questions were considered non-experimenters.

Table 3 shows the prevalence of smoking experimentation by MVPA status. Smoking experimentation did not differ significantly by MVPA $(p=0.159)$. Among the non-experimenters, $28.3 \%$ completed adequate MVPA compared to $32.9 \%$ of experimenters. Further analyses examining mean differences in the demographic and psychosocial correlates by the four-level categorical variable yielded statistically significant results, which are presented in Tables 4 and $\mathbf{5}$. Regardless of smoking experimentation status, a higher proportion of boys, compared to girls, completed adequate MVPA $(p<0.001)$. Among those with inadequate MVPA, a higher proportion of boys (18.7\%) compared to girls $(11.4 \%)$ had experimented with cigarettes $(p<0.001)$. Girls were more likely than boys to neither have experimented with cigarettes nor complete adequate MVPA (66.2\% vs. $45.0 \%)$. Regardless of level of MVPA, experimenters were older than non-experimenters $(p<0.001)$. Regardless of smoking status, there were no significant differences in age between those with adequate and inadequate MVPA ( $p=0.672$; data not shown).

In Table 5, we present gender- and age-adjusted means for the psychosocial correlates by the fourlevel categorical variable. Irrespective of MVPA level, experimenters reported more parents who smoked, and more friends who smoked, compared to non-experimenters ( $p<0.05$ for all). Similarly, at both levels of MVPA, experimenters held more favorable outcome expectations (in other words, perceived more social benefits from smoking) associated with smoking than nonexperimenters did $(p<0.05)$ and more participants who had experimented with cigarettes had also 
Table 3: Prevalence of Smoking Experimentation by Moderate-to-Vigorous Physical Activity $(\mathrm{N}=1,098)$

\begin{tabular}{|ccccc|}
\hline \multirow{2}{*}{ MVPA } & \multicolumn{2}{c}{ Smoking Status } & \multirow{2}{*}{ Total } & p-value \\
\cline { 2 - 4 } & Non-Experimenter & $\begin{array}{c}\text { Experimenter } \\
\mathbf{N}(\%)\end{array}$ & & \\
\hline Inadequate & $611(71.7)$ & $165(67.1)$ & $776(70.7)$ & \\
Adequate & $241(28.3)$ & $81(32.9)$ & $322(29.3)$ & 0.159 \\
Total & $852(77.6)$ & $246(22.4)$ & 1,098 & \\
\hline
\end{tabular}

Participants who reported being physically active for at least 60 minutes on at least 5 days were considered to have met requirements; all others were considered to not have met requirements. Participants who responded yes to either "Have you ever smoked a whole cigarette?" or "Have you ever tried a cigarette, even a puff?" were considered experimenters; participants who answered no to both questions were considered non-experimenters.

Table 4: Participant Characteristics by Moderate-to-Vigorous Physical Activity and Smoking Experimentation Status $(\mathrm{N}=1,098)$

\begin{tabular}{|c|c|c|c|c|c|}
\hline \multirow[b]{2}{*}{ Demographics } & \multicolumn{4}{|c|}{ MVPA and Smoking Experimentation Status } & \multirow[b]{2}{*}{ p-value } \\
\hline & $\begin{array}{c}\text { Adequate MVPA \& } \\
\text { Non-Experimenter } \\
\text { N (\%) }\end{array}$ & $\begin{array}{c}\text { Adequate MVPA } \\
\text { \& Experimenter } \\
\text { N (\%) }\end{array}$ & $\begin{array}{c}\text { Inadequate MVPA \& } \\
\text { Non-Experimenter } \\
\text { N (\%) }\end{array}$ & $\begin{array}{c}\text { Inadequate MVPA } \\
\text { \& Experimenter } \\
\text { N (\%) }\end{array}$ & \\
\hline Overall & $241(21.9)$ & $81(7.4)$ & $611(55.7)$ & $165(15.0)$ & \\
\hline Gender & & & & & $<0.001$ \\
\hline Female & $100(18.1)$ & $24(4.3)$ & $366(66.2)$ & $63(11.4)$ & \\
\hline Male & $141(25.9)$ & $57(10.5)$ & $245(45.0)$ & $102(18.7)$ & \\
\hline Age (Years) & & & & & $<0.001$ \\
\hline Mean (SE) & $14.15(1.03)^{\mathrm{a}}$ & $14.74(0.92)^{b}$ & $14.16(1.00)^{a}$ & $14.95(0.98)^{b}$ & \\
\hline Country of Birth & & & & & 0.059 \\
\hline Mexico & $69(24.1)$ & $22(7.7)$ & $166(58.0)$ & $29(10.1)$ & \\
\hline US & $172(21.2)$ & $59(7.3)$ & $445(54.8)$ & $136(16.7)$ & \\
\hline Parental Education & & & & & 0.739 \\
\hline$<\mathrm{HS}$ & $150(20.9)$ & $48(6.7)$ & $406(56.6)$ & $113(15.8)$ & \\
\hline $\mathrm{HS}$ & $45(23.4)$ & $16(8.3)$ & $104(54.2)$ & $27(14.1)$ & \\
\hline$>\mathrm{HS}$ & $46(24.3)$ & $17(9.0)$ & $101(53.4)$ & $25(13.2)$ & \\
\hline
\end{tabular}

Means with the same letters are not statistically significantly different from each other. Conversely, means with different letters are statically significantly different from each other.

used alcohol compared to those who had not smoked $(p<0.05)$.

Regarding sensation-seeking tendencies, experimenters, regardless of MVPA level, had higher scores on drug and alcohol seeking and social disinhibition than non-experimenters $(p<0.05$ for all). Thrill and adventure-seeking scores were highest among participants who both completed adequate MVPA and had experimented $(p<0.05)$ compared to the other groups. Similarly, among those with inadequate MVPA, experimenters reported higher levels of thrill and adventure seeking than non-experimenters $(p<0.05)$. Experi- menters had higher anxiety scores than nonexperimenters, regardless of their MVPA levels $(p<0.05)$. Those who reported adequate MVPA, regardless of experimenter status, reported lower anxiety compared to those who were less active $(p<0.05)$.

Regardless of smoking status, participants with adequate MVPA participated in a higher number of both school and community sports teams than those who did not ( $p<0.05$ for both). Experimenters with inadequate MVPA reported lower SSS than non-experimenters irrespective of MVPA level ( $p<0.05$ for both) and reported more body 
Table 5: Psychosocial Risk Factors by Moderate-to-Vigorous Physical Activity and Smoking Experimentation Status $(\mathrm{N}=1,098)$

\begin{tabular}{|c|c|c|c|c|c|}
\hline \multirow[b]{2}{*}{ Correlate } & \multicolumn{4}{|c|}{ MVPA and Smoking Experimentation Status } & \multirow[b]{2}{*}{ p-value } \\
\hline & $\begin{array}{l}\text { Adequate } \\
\text { MVPA \& Non- } \\
\text { experimenter } \\
\text { Mean (SE) }\end{array}$ & $\begin{array}{c}\text { Adequate } \\
\text { MVPA \& } \\
\text { Experimenter } \\
\text { Mean (SE) }\end{array}$ & $\begin{array}{l}\text { Inadequate } \\
\text { MVPA \& Non- } \\
\text { experimenter } \\
\text { Mean (SE) }\end{array}$ & $\begin{array}{l}\text { Inadequate } \\
\text { MVPA \& } \\
\text { Experimenter } \\
\text { Mean (SE) }\end{array}$ & \\
\hline No. parents who smoke & $0.33(0.04)^{a}$ & $0.54(0.06)^{b}$ & $0.32(0.02)^{a}$ & $0.51(0.05)^{b}$ & $<0.001$ \\
\hline No. peers who smoke & $0.27(0.04)^{a}$ & $0.85(0.08)^{b}$ & $0.19(0.03)^{a}$ & $0.76(0.06)^{b}$ & $<0.001$ \\
\hline Outcome Expectations & $1.22(0.03)^{\mathrm{a}}$ & $1.65(0.05)^{b}$ & $1.25(0.02)^{a}$ & $1.65(0.04)^{b}$ & $<0.001$ \\
\hline Alcohol Use & $0.23(0.03)^{a}$ & $0.57(0.05)^{b}$ & $0.19(0.02)^{a}$ & $0.61(0.03)^{b}$ & $<0.001$ \\
\hline Drug \& Alcohol Seeking & $0.89(0.09)^{a}$ & $2.16(0.16)^{b}$ & $0.79(0.06)^{a}$ & $2.26(0.11)^{b}$ & $<0.001$ \\
\hline Social Disinhibition & $3.27(0.12)^{a}$ & $4.19(0.20)^{b}$ & $2.93(0.07)^{a}$ & $4.06(0.15)^{b}$ & $<0.001$ \\
\hline Thrill \& Adventure Seeking & $6.97(0.20)^{\mathrm{a}, \mathrm{c}}$ & $8.56(0.34)^{b}$ & $6.44(0.12)^{a}$ & $7.36(0.24)^{\mathrm{a}, \mathrm{c}}$ & $<0.001$ \\
\hline Trait Anxiety & $36.85(0.60)^{a}$ & $40.50(1.04)^{\mathrm{b}, \mathrm{c}}$ & $37.66(0.38)^{a, c}$ & $41.85(0.74)^{b}$ & $<0.001$ \\
\hline Community Team Sports & $2.20(0.10)^{a}$ & $2.06(0.18)^{a}$ & $1.18(0.07)^{b}$ & $1.30(0.13)^{b}$ & $<0.001$ \\
\hline School Team Sports & $2.21(0.11)^{a}$ & $2.49(0.19)^{a}$ & $1.44(0.07)^{b}$ & $1.58(0.13)^{b}$ & $<0.001$ \\
\hline Subjective Social Status & $7.95(0.09)^{a}$ & $7.63(0.16)^{a, b}$ & $7.85(0.06)^{a}$ & $7.17(0.12)^{a, b}$ & $<0.001$ \\
\hline Body Image & $1.58(0.14)^{a}$ & $2.04(0.25)^{a, b}$ & $1.76(0.09)^{\mathrm{a}}$ & $2.32(0.18)^{a, b}$ & 0.009 \\
\hline Acculturation & $3.52(0.05)^{a}$ & $3.49(0.08)^{a}$ & $3.49(0.03)^{a}$ & $3.52(0.06)^{a}$ & 0.921 \\
\hline BMI & $24.75(0.41)^{a}$ & $25.35(0.71)^{a}$ & $25.05(0.26)^{a}$ & $25.29(0.51)^{a}$ & 0.819 \\
\hline
\end{tabular}

SE: standard error. Means with the same letters are not statistically significantly different from each other. Conversely, means with different letters are statically significantly different from each other. Higher scores on the following scales reflect a stronger endorsement of the attribute assessed: positive outcome expectations from smoking, anxiety, subjective social status, body image concerns, drug and alcohol seeking, social disinhibition, thrill and adventure seeking.

image concerns than non-experimenters, regardless of MVPA level $(p<0.05)$.

Finally, we found neither acculturation nor BMI was significantly associated with the interaction of MVPA and smoking experimentation.

\section{DISCUSSION}

This study examined differences in smoking and physical activity-related psychosocial factors among four groups of Mexican origin adolescents stratified by combined physical activity and smoking-experimenter status. In our overall sample, $22.4 \%$ had experimented with cigarettes, and $29.3 \%$ reported adequate MVPA. Consistent with our previous research, boys were more likely than girls to complete adequate MVPA and experiment with cigarettes [10]. Among our Mexican origin participants, smoking experimentation rates did not differ significantly by level of MVPA, although more experimenters compared to non-experimenters completed adequate MVPA (33\% vs. $28 \%$, respectively).
Our results suggest that, with the exception of acculturation and BMI, the factors we examined differed significantly across the four groups of physical activity and smoking status. The most significant differences appear to be driven by smoking or physical activity, rather than a combination of the two. For example, as would be expected, completing adequate physical activity was associated with greater involvement in community and school sports [28]. Patterns in the remainder of the factors across groups suggest that smoking status, as opposed to physical activity, might be the main driving differentiator. For example, also as expected, experimenters reported higher means for parental and peer smoking influence, regardless of level of MVPA [29]. We did not observe differences in MVPA at either level of experimentation. A similar pattern was found for alcohol use, smoking expectations, as well as the drug and alcohol-seeking scales and social disinhibition sensation-seeking scales. All four factors have been found in previous work to be associ- 
ated with tobacco use in this population $[9,10$, 12,13].

Thrill and adventure seeking, much like the other sensation-seeking scales, differed by smoking and MVPA status. However, the highest levels of thrill and adventure seeking were in experimenters who completed adequate MVPA. This finding parallels previous research in which those with high thrill and adventure-seeking scores were more likely to express their sensation-seeking tendencies across a variety of behaviors, including physical activity and smoking [10,30].

For both SSS and body image, those who engaged in smoking experimentation and inadequate MVPA perceived they were lower on the social ladder and had a poorer body image compared to non-experimenters. These findings support previous research that suggests an association between smoking and low social status, both in the sample of our study and in other populations of adolescents [9,31]. They also parallel conclusions of previous studies in which poor body image was associated with smoking uptake and continuation, possibly as a means of weight control [11,32]. For body image particularly, it is notable that the mean for experimenters who completed adequate MVPA did not differ significantly from the other 3 groups. This suggests that among Mexican origin youth MVPA might not be a behavior used to ameliorate poor body image to the degree that it is used in other populations of adolescents [33].

Anxiety scores were higher among experimenters with inadequate levels of MVPA compared to nonexperimenters, irrespective of their MVPA status. Among those with adequate MVPA, experimenters reported higher anxiety levels compared to non-experimenters. Together, these findings underscore the possibility that because anxiety levels are higher among experimenters than nonexperimenters, smoking, more so than physical activity, is a behavior Mexican origin adolescents use to cope with anxiety. However, further research designed to decipher either active or subconscious choice in engaging in either behavior as a coping mechanism is needed to draw such a conclusion.
Overall, findings suggest that with the exception of team sports participation, differences among the variables examined in our study are driven primarily by smoking behavior. This finding is not unexpected, given that three of the variables we examined specifically focus on smoking (social influence and outcome expectations) and the other psychosocial factors have been found to be associated with smoking in previous studies. Thus among participants of the same smoking status, physical activity does not appear to confer notable protection from experimentation against the impact of peer and parental influence associated with smoking or positive smoking expectations.

Our findings also suggest that certain aspects of sensation tendencies, such as drug and alcohol sensation seeking, are more readily expressed through smoking than through physical activity, and that the latter insufficiently protects against the former. However, the highest levels of thrill and adventure seeking were found in physically active experimenters. Rodriguez et al. suggested that an adolescent's choice to engage in MVPA may be more a function of the immediate feeling roused by the activity, and less about an awareness of long-term benefits [8]. Thus, our results suggest among youth with high levels of thrill and adventure seeking, targeting interventions that promote physical activity as an alternative to smoking, could be an effective strategy. Of importance, among those with scores in the highest quartile of thrill and adventure seeking, $82 \%$ have experimented and $69 \%$ complete adequate MVPA (data not shown).

It is noteworthy that engaging in adequate MVPA does not appear to confer notable protection from experimentation against psychosocial factors associated with both behaviors including anxiety, low SSS, and poor body image. Our previous research suggests that among Mexican origin youth, a similar motivation might be in play for smoking, particularly as it relates to expression of coping with anxiety, poor body image, or low SSS [9-11]. Our findings in this study, however, suggest that the appeal of smoking might be stronger than that of physical activity for those with these less desirable psychosocial characteristics. Given that these same risk factors are associated with cur- 
rent smoking and low levels of physical activity among adults our results underscore the possibility that adolescents with these characteristics may be particularly vulnerable for a lifetime of smoking and physical inactivity [34-39]. Additional research is warranted to determine whether it would be beneficial to tailor interventions to address both deleterious behaviors simultaneously.

Our study has methodological strengths. First, our sample came from a population-based cohort, representing an ethnically homogenous and predominantly low-income population of Mexican origin youth. This population is largely understudied and underserved. Furthermore, given the sensitive nature of some of the questions, it is also a strength that all data were collected using PDAs, which ensured that participants read and answered the questions without concern for their parents hearing or seeing their responses.

Our study has some limitations. For one, participants in our sample were all of Mexican origin. The homogeneity of our sample is a limitation in that it hinders the generalizability of our conclusions to other demographics, including other Hispanic youth from different countries of origin. Furthermore, smoking and alcohol-use were assessed via self-report and unverified using biological samples or other methods of crossvalidation. Therefore, smoking and alcohol-use may be underreported. However, use of the PDA for data collection provided a secure non-invasive approach, away from parental eyes for reporting purposes. Evidence suggests, however, that the validity of self-reported data tends to increase when participants believe biological samples may be requested, which was the case in our study (saliva was collected for genetic analysis) [40]. Finally, while we examined social influence from family and friends regarding smoking behavior, we did not do so regarding physical activity. Given the important role of social influence on behavior and the importance of the family in the Mexican culture, it would be valuable to examine the role of social influence on physical activity in order to determine the relative influence of each.

Overall, our results have implications for smoking prevention and the promotion of physical activity among youth of Mexican origin. They highlight an opportunity to address physical activity as an alternative to smoking among youth with high sensation-seeking tendencies and to engage those with less desirable psychosocial characteristics, such as low SSS, anxiety, and/or more body image concerns in health promotion activities to address both deleterious behaviors. By gaining a clearer understanding as to why Mexican origin youth do not engage in adequate MVPA and/or do smoke, future interventions can be designed to promote physical activity as capable of addressing some of those motivations. Based on our findings, such interventions would be particularly impactful if they targeted thrill and adventure seekers and addressed the sensation seeking-related motivations for smoking while promoting physical activity as an alternative. In addition, further research examining a broader range of risk factors that potentially relate to both behaviors, as well as appropriate messaging associating the benefits of MVPA with the appeal of smoking, is needed to further tailor such interventions.

\section{ACKNOWLEDGEMENTS}

We thank the cohort staff for conducting all field interviews and maintaining the high participation rates. We thank the participants for providing the data and their parents for permitting their children to join the study. Without their support this research would not be possible. This research is supported by the National Cancer Institute grants [CA105203 to MRS, and CA126988 to AVW]; funds collected pursuant to the Comprehensive Tobacco Settlement of 1998, and appropriated by the 76th legislature to The University of Texas $M$. D. Anderson Cancer Center; by the Caroline W. Law Fund for Cancer Prevention, and by the Dan Duncan Family Institute for Cancer Prevention and Risk Assessment. The funders did not contribute to the design and conduct of the study, the data collection, analysis, and interpretation of the data, the preparation, review, or approval of the manuscript.

\section{REFERENCES}

[1] Substance Abuse and Mental Health Services Administration (SAMHSA). National survey on drug use and health. Washington D.C.; 2009 [accessed 01/30/2014]. Available from http://oas. samhsa.gov/nsduh.htm\#NSDUHinfo. 
[2] United States Department of Health and Human Services. Preventing tobacco use among youth and young adults: A report of the Surgeon General, 2012. Georgia; 2012 [accessed 01/30/2014]. Available from http://www.surgeongeneral.gov/ library/reports/preventing-youth-tobacco-use/fullreport.pdf.

[3] Sallis J. Age related decline in physical activity: A synthesis of human and animal studies. Med Sci Sports Exerc 2000; 32: 1598-1600.

[4] Brodersen N H, Steptoe A, Boniface DR, Wardle J. Trends in physical activity and sedentary behavior in adolescence: Ethnic and socioeconomic differences. Br J Sports Med 2007; 41: 140-4.

[5] Kelder SH, Perry CL, Klepp KI, Lytle LL. Longitudinal tracking of adolescent smoking, physical activity, and food choice behaviors. Am J Public Health 1994; 84: 1121-6.

[6] Paavola M, Vartiainen E, Haukkala A. Smoking, alcohol use, and physical activity: A 13-year longitudinal study ranging from adolescence into adulthood. J Adolesc Health 2004; 35: 238-44.

[7] Audrain-McGovern J, Rodriguez D, Wileyto EP, Schmits KH, Shields PG. Effect of team sport participation on genetic predisposition to adolescent smoking progression. Arch Gen Psychiatry 2006; 63: 433-41.

[8] Rodriguez D, Dunton GF, Tscherne J, Sass J. Physical activity and adolescent smoking: A moderated mediation model. Ment Health Phys Act 2008; 1: 17-25.

[9] Wilkinson AV, Shete S, Vasudevan V, Prokhorov $A V$, Bondy ML, Spitz MR. The influence of subjective social status on the relationship between positive outcome expectations and experimentation with cigarettes. J Adolesc Health 2009; 44: 342-8.

[10] Wilkinson AV, Okeke NL, Springer AE, et al. Experimenting with cigarettes and physical activity among Mexican origin youth: A cross-sectional analysis of the interdependent associations among sensation seeking, acculturation, and gender. BMC Public Health 2012; 12: 332.

[11] Okeke NL, Forman MR, Spitz MR, Wilkinson AV. The associations of body image, anxiety, and smoking among Mexican-origin youth. J Adolesc Health 2013; 53: 209-14.

[12] Torabi MR, Bailey MJ, Majd-Jabbari M. Cigarette smoking as a predictor of alcohol and other drug use by children and adolescents: Evidence of the 'gateway drug effect'. J Sch Health1993; 63: 3026.

[13] Chen X, Unger JB, Palmer P, et al. Prior cigarette smoking initiation predicting current alcohol use: Evidence for a gateway drug effect among California adolescents from eleven ethnic groups. Addict Behav 2002; 27: 799-817.
[14] Wilkinson AV, Gabriel KP, Wang J, et al. Sensation-seeking genes and physical activity in youth. Genes Brain and Behav 2012; 12: 181-8.

[15] Miller E. Associations of perceived body image and subjective social status with levels of physical activity in Mexican American adolescents [unpublished master's thesis]. University of Texas Health Science Center at Houston; 2013.

[16] Ennis SR, Rios-Vargas M, Albert NG. The Hispanic Population: 2010. U.S. Census Bureau. Report number: C2010BR-04, 2011.

[17] Humes KR, Jones NA, Ramirez RR. Overview of Race and Hispanic Origin: 2010. U.S. Census Bureau. Report number: C2010BR-02, 2011.

[18] Logan JR, Turner RN. Hispanics in the United States: Not only Mexicans. Brown University. Report number: 03202013, 2013.

[19] Centers for Disease Control and Prevention (CDC). 2011 Texas Youth Risk Behavior Survey results. Georgia: 2012 [accessed 01/30/2014]. Available from http://www.dshs.state.tx.us/chs/ yrbs/query/yrbss_form.shtm.

[20] Wilkinson AV, Waters AJ, Vasudevan V, Bondy ML, Prokhorov AV, Spitz MR. Correlates of susceptibility to smoking among Mexican origin youth residing in Houston, Texas: A crosssectional analysis. BMC Public Health 2008; 8: 337.

[21] Spelman AR, Spitz MR, Kelder SH, et al. Cognitive susceptibility to smoking: Two paths to experimenting among Mexican origin youth. Cancer Epidemiol Biomarkers Prev 2009; 18: 3459-67.

[22] Horn K, Geri D, Branstetter SA, et al. Effects of physical activity on teen smoking cessation. Pediatrics 2011; 128: e801-e811.

[23] Ussher M, West R, McEwen A, Taylor A, Steptoe A. Randomized controlled trial of physical activity counseling as an aid to smoking cessation: 12 month follow-up. Addict Behav 2007; 32: 3060-4.

[24] Saunders KM. Evaluation of recruitment strategies for obtaining a representative cohort of urban Mexican Americans [unpublished master's thesis]. University of Texas Health Science Center at Houston; 2005.

[25] Wilkinson AV, Spitz MR, Strom SS, Prokhorov AV, Barcenas $\mathrm{CH}$, Cao Y, Saunders KC, Bondy LM. Effects of nativity, age at migration, and acculturation on smoking among adult Houston residents of Mexican descent. Am J Public Health 2005; 95: 1043-1049.

[26] Centers for Disease Control and Prevention (CDC). 2005 Youth Risk Behavior survey. Georgia: 2006 [accessed 01/30/2014]. Available from $\mathrm{ftp}: / / \mathrm{ftp} . c d c . g o v / p u b / d a t a / y r b s / 2005 / Y R B S \_2005$ _National_User_Guide.pdf. 
[27] Backlund E, Sorlie, PD, Johnson NJ. A comparison of the relationships of education and income with mortality: the national longitudinal mortality study. Soc Sci Med 1999; 49: 1373-1384.

[28] Ekelund U, Tomkinson G, Armstrong N. What proportion of youth are physically active: Measurement issues and recent time trends. $\mathrm{Br} J$ Sports Med 2011; 45: 859-65.

[29] Vitória PD, Salqueiro MF, Silva SA, de Vries H. Social influence, intention to smoke, and adolescent smoking behavior longitudinal relations. $\mathrm{Br} \mathrm{J}$ Health Psychol 2011; 16: 779-98.

[30] Greene K, Krcmar M, Walters LH, Rubin DL, Hale J, Hale L. Targeting adolescent risk-taking behaviors: The contributions of egocentrism and sensation-seeking. J Adolesc 2000; 23: 439-61.

[31] Finkelstein DM, Kubzansky LD, Goodman E. Social status, stress and adolescent smoking. J Adolesc Health 2006; 39: 678-85.

[32] Dowdell EB, Santucci ME. Health Risk Behavior Assessment: Nutrition, Weight, and Tobacco Use in One Urban Seventh-grade Class. Public Health Nursing 2004; 21: 128-136.

[33] Kirkcaldy, BD, Shephard RJ, Siefen RG. The relationship between physical activity and selfimage and problem behaviour among adolescents. Social Psychiatry and Psychiatr Epidemiol 2002;37:554-550.

[34] Buckner J, Vinci D. Smoking and social anxiety: The roles of gender and smoking motives. Addict Behav 2013; 38: 2388-91.

[35] Clark MM, Croghan IT, Reading S, et al. The relationship of body image dissatisfaction to cigarette smoking in college students. Body Image 2005; 2: 263-70.

[36] Reitzel, LR, Businelle MS, Kendzor DE, et al. Subjective social status predicts long-term smoking abstinence. BMC Public Health 2011; 11: 135.

[37] Anderson E, Shivakumar G. Effects of exercise and physical activity on anxiety. Front Psychiatry 2013; 4: 27.

[38] LePage ML, Crowther JH. The effects of exercise on body satisfaction and affect. Body Image 2010; 7: 124-30.
[39] Ghaed, SG, Gallo LC. Subjective social status, objective socioeconomic status, and cardiovascular risk in women. Health Psychol 2007; 26: 668-74.

[40] Murray DM, O'Connell CM, Schmid LA, Perry $C L$. The validity of smoking self-reports by adolescents: A reexamination of the bogus pipeline effect. Addict Behav 1987; 12: 7-15.

[41] Norris AE, Ford K, Bova CA. Psychometrics of a brief acculturation scale for Hispanics in a probability sample of urban Hispanic adolescents and young adults. Hisp J Behav Sci 1996; 18: 29-38.

[42] Dalton MA, Sargent JD, Beach ML, Bernhardt AM, Stevens M. Positive and negative outcome expectations of smoking: Implications for prevention. Prev Med 1999; 29: 460-5.

[43] Bandura A. Social foundations of thought and action: A social cognitive theory. Englewoord Cliffs, NJ: Prentice-Hall; 1986.

[44] Spielberger CD. Assessment of state and trait anxiety: Conceptual and methodological issues. South Psych 1985; 2: 6-16.

[45] Spielberger CD, Gonzalez-Reigosa F, MartinezUrrutia A, Natalicio LD, Natalicio, DS. Development of the Spanish edition of the state-trait anxiety inventory. Interam J Psychol 1971; 5: 145-58.

[46] Goodman E, Adler NE, Kawachi I, Frazier AL, Huang B, Colditz GA. Adolescents' perceptions of social status: Development and evaluation of a new indicator. Pediatrics 2011; 108: e31.

[47] Cooper PJ, Taylor MJ, Cooper Z, Fairbum CG. The development and validation of the Body Shape Questionnaire. J Eat Disord 1987; 6: 48594.

[48] Russo MF, Stokes GS, Lahey BB, et al. A sensation seeking scale for children: Further refinement and psychometric development. J Psychopathol Behav Assess 1993; 15: 69-86.

[49] Zuckerman M. Sensation Seeking and Risky Behavior. Washington, DC: APA; 2007. 